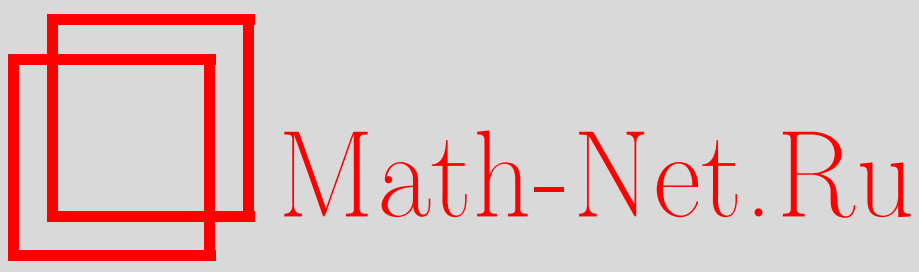

В. Г. Михайлов, Пуассоновская аппроксимация для распределения числа появлений заданной цепочки знаков в выходной последовательности генератора Пола, Матем. вопр. криптогр., 2014, том 5, выпуск 4, 63-71

DOI: https://doi.org/10.4213/mvk135

Использование Общероссийского математического портала Math-Net.Ru подразумевает, что вы прочитали и согласны с пользовательским соглашением

http://www. mathnet.ru/rus/agreement

Параметры загрузки:

IP: 3.80 .253 .173

26 апреля 2023 г., 04:23:58

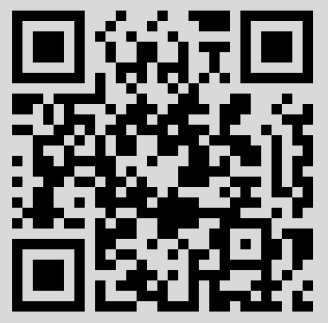


МАТЕМАТИЧЕСКИЕ ВОПРОСЫ КРИПТОГРАФИИ

2014 T. 5 № 4 C. 63-71

УДК 519.212.2

\section{Пуассоновская аппроксимация для распределения числа появлений заданной цепочки знаков в выходной последовательности генератора Пола}

\section{В. Г. Михайлов}

Математический институт им. В.А. Стеклова РАН, Москва

Получено 14.I.2014

Доказана предельная теорема Пуассона (с оценками точности аппроксимации) для распределения числа появлений в выходной последовательности генератора Пола заданной не допускающей самоналожения цепочки знаков.

Ключевые слова: генератор Пола, пуассоновская аппроксимация, метода Чена - Стейна.

Poisson approximation for the distribution of the frequency of a given pattern in the outcome sequence of the MCV-generator V. G. Mikhailov

Steklov Mathematical Institute of RAS, Moscow

Abstract. Poisson limit theorem for the distribution of the number of occurrences of a given non-overlapping pattern in the output sequence of the MCV-generator is proved along with the estimate of the convergence rate.

Keywords: the MCV-generator, Poisson approximation, the Chen - Stein method.

Citation: Mathematical Aspects of Cryptography, 2014, vol. 5, no. 4, pp. 63-71 (Russian)

(C)2014 В. Г. Михайлов 


\section{1. Введение}

Генератор Пола (см. [1]) состоит из $r$ циклических регистров сдвига взаимно простых длин $m_{1}, \ldots, m_{r}$ над кольцом вычетов по некоторому модулю $M$. Обозначим через $\left(x_{0}^{(k)}, \ldots, x_{m_{k}-1}^{(k)}\right), k=1, \ldots, r$, заполнения регистров генератора. Условимся использовать в индексах обозначение $t(m)$ для наименьшего неотрицательного вычета числа $t$ по модулю $m$. Знаки выходной последовательности генератора образуются по формуле

$$
z_{t}=x_{t\left(m_{1}\right)}^{(1)}+\ldots+x_{t\left(m_{r}\right)}^{(r)} \bmod M .
$$

Если заполнения регистров являются независимыми случайными величинами $X_{i}^{(k)}$, распределенными равномерно на множестве $\{0, \ldots, M-$ $1\}$, то выходная последовательность $Z_{t}$ является случайной и имеет период длины $L=m_{1} \cdot \ldots \cdot m_{r}$. Очевидно, что распределение отрезка выходной последовательности длины $T \leq \max \left\{m_{1}, \ldots, m_{r}\right\}$ совпадает с распределением такого же отрезка независимых случайных величин с равномерным распределением. Считается (см. [1]), что при длине выходной последовательности генератора Пола не больше $2 \sqrt{L}$ эта последовательность в значительной степени обладает свойствами равновероятной случайной последовательности. При существенно больших длинах отрезок выходной последовательности генератора со случайным заполнением регистров по своим вероятностным свойствам заметно отличается от отрезка равновероятной последовательности. Так, в [2] было показано, что при $M=2$ распределение числа единиц на полном цикле длины $L$ выходной последовательности генератора не является биномиальным и при неограниченном увеличении длин регистров и/или числа регистров сходится к распределениям, отличным от нормального. Там же были получены оценки скорости сходимости в соответствующих предельных теоремах.

В настоящей заметке показывается, что наряду с этим любой отрезок выходной последовательности генератора со случайными равновероятными заполнениями обладает следующим важным свойством равновероятной случайной последовательности. Пусть задана некоторая цепочка (слово) $\left(a_{1}, \ldots, a_{s}\right)$ элементов множества $\{0, \ldots, M-1\}$, удовлетворяющая условиям

$$
a_{1} \neq a_{s},\left(a_{1}, a_{2}\right) \neq\left(a_{s-1}, a_{s}\right), \ldots,\left(a_{1}, \ldots, a_{s-1}\right) \neq\left(a_{2}, \ldots, a_{s}\right) .
$$

Другими словами, цепочка $\left(a_{1}, \ldots, a_{s}\right)$ не допускает частичного самоналожения.

В работе показано, что для распределения числа появлений в отрезке выходной последовательности конкретной цепочки, не допускающей 
самоналожения, справедлива оценка точности пуассоновской аппроксимации, аналогичная оценке для распределения числа появлений такой же цепочки в последовательности независимых случайных величин, распределенных равномерно на алфавите. Эта оценка содержательна для любых достаточно длинных отрезков выходной последовательности, в том числе и для ее полного периода. Из этой оценки в работе выводятся пуассоновская и «околопуассоновская» нормальная предельные теоремы, аналогичные классическим теоремам для числа появлений цепочки, не допускающей самоналожения (см., например, [3]).

Естественным является предположение о том, что аналогичными асимптотическими свойствами обладают распределения и многих других классов редких событий в выходной последовательности генератора Пола.

\section{2. Формулировки результатов}

Рассматривается генератор с $r$ регистрами взаимно простых длин $m_{1}, \ldots, m_{r}$. Пусть $X^{(i)}=\left(X_{0}^{(i)}, \ldots, X_{m_{i}-1}^{(i)}\right)$ - случайное заполнение $i$-го регистра, $i=1, \ldots, r$. Все случайные величины $X_{j}^{(i)}$ в этих наборах независимы в совокупности и имеют равномерное распределение на множестве $\{0, \ldots, M-1\}$. Выходная последовательность генератора задается формулой

$$
Z_{t}=X_{t\left(m_{1}\right)}^{(1)}+\ldots+X_{t\left(m_{r}\right)}^{(r)} \bmod M
$$

Рассмотрим некоторую цепочку $a_{1}, \ldots, a_{s}$ из элементов множества $\{0, \ldots, M-1\}$, удовлетворяющую условию (2). Появлением в последовательности $\left\{Z_{t}\right\}$ цепочки $a_{1}, \ldots, a_{s}$ с началом в точке $t$ естественно считать событие

$$
\left\{X_{t}=a_{1}, \ldots, X_{t+s-1}=a_{s}\right\} \text {. }
$$

Нас интересует распределение числа $\xi(s, T)$ тех появлений цепочки $a_{1}, \ldots, a_{s}$, начала которых лежат в заданном отрезке выходной последовательности длины $T \leq m_{1} \ldots m_{r}$. В обозначении случайной величины $\xi(s, T)$ не указаны ни расположение рассматриваемого отрезка, ни выбор знаков $a_{1}, \ldots, a_{s}$, поскольку распределение величины $\xi(s, T)$ от этих параметров не зависит.

Условимся использовать обозначение $\mathcal{L}(W)$ для распределения случайной величины $W$ и обозначение $\operatorname{Po}(\lambda)$ для распределения Пуассона с параметром $\lambda$. Напомним, что расстояние по вариации между распределениями случайных величин $U$ и $V$ на множестве неотрицательных 
целых чисел выражается формулой

$$
\rho(\mathcal{L}(U), \mathcal{L}(V))=\frac{1}{2} \sum_{k=0}^{\infty}|\mathbf{P}\{U=k\}-\mathbf{P}\{V=k\}| .
$$

Положим

$$
\Lambda=T M^{-s} .
$$

Если $s<\min \left\{m_{1}, \ldots, m_{r}\right\}$, то $\Lambda=\mathbf{E} \xi(s, T)$.

Будем использовать обозначение $] b[$ для наименьшего целого числа, превосходящего число $b$ или равного ему.

Теорема 1. Пусть $M \geq 2,2 s-1 \leq \min \left\{m_{1}, \ldots, m_{r}\right\}, T \leq m_{1} \ldots m_{r}$. Тогда

$$
\rho(\mathcal{L}(\xi(s, T)), \operatorname{Po}(\Lambda)) \leq \frac{(M+1)(2 s-1)}{M^{s}}(] \frac{T}{m_{1}}[+\ldots+] \frac{T}{m_{r}}[) .
$$

Нетрудно показать, что оценка (5) грубее аналогичной оценки для распределения числа $\eta(s, T)$ появлений цепочки $a_{1}, \ldots, a_{s}$ в последовательности из $T$ независимых случайных величин, распределенных равномерно на множестве $\{0, \ldots, M-1\}$. Для распределения $\eta(s, T)$ аналогично (5) выводится неравенство

$$
\rho(\mathcal{L}(\eta(s, T)), \operatorname{Po}(\Lambda)) \leq \frac{(M+1)(2 s-1)}{M^{s}} .
$$

Следствие 1. Пусть $M \geq 2, T \leq m_{1} \ldots m_{r}, a m_{1}, \ldots, m_{r}, s, T \rightarrow \infty$ maк, что $\Lambda=T M^{-s} \rightarrow \lambda \in(0, \infty) u$

$$
\max _{i, j}\left(\frac{\ln m_{i}}{m_{j}}\right) \rightarrow 0 .
$$

Тогда распределение случайной величины $\xi(s, T)$ сходится $к$ распределению Пуассона с параметром $\lambda$.

Следствие 2. Пусть $M \geq 2, T \leq m_{1} \ldots m_{r}$, a $m_{1}, \ldots, m_{r}, s, T \rightarrow \infty$ maк, что $\Lambda=T M^{-s} \rightarrow \infty u$

$$
\frac{T}{M^{s}} \max _{i, j}\left(\frac{\ln m_{i}}{m_{j}}\right) \rightarrow 0 .
$$

Тогда распределение случайной величины $(\xi(s, T)-\Lambda) \Lambda^{-1 / 2}$ сходится $\kappa$ стандартному нормальному распределению. 


\section{3. Доказательства}

Доказательство теоремы 1. Введем случайные события

$$
E_{t}(a)=\left\{Z_{t}=a\right\}=\left\{X_{t\left(m_{1}\right)}^{(1)}+\ldots+X_{t\left(m_{r}\right)}^{(r)} \equiv a \bmod M\right\} .
$$

Набор значений $i_{1}=t\left(m_{1}\right), \ldots, i_{r}=t\left(m_{r}\right)$ и условие $0 \leq t<m_{1} \ldots m_{r}$ однозначно определяют величину $t$. Поэтому для индикатора появления цепочки $a_{1}, \ldots, a_{s}$ в момент $t$ можно использовать обозначение

$$
W\left(i_{1}, \ldots, i_{r}\right)=I\left\{E_{t}\left(a_{1}\right) \cap \ldots \cap E_{t+s-1}\left(a_{s}\right)\right\},
$$

где $I\{E\}-$ индикатор случайного события $E$.

Введем множество

$$
\Gamma(T)=\left\{\left(t\left(m_{1}\right), \ldots, t\left(m_{r}\right)\right): t=0, \ldots, T-1\right\} .
$$

Используя тот факт, что распределение случайной величины $\xi(s, T)$ не зависит от расположения отрезка наблюдений, заключаем, что задача свелась к изучению распределения случайной величины

$$
\xi^{\prime}(s, T)=\sum_{\left(i_{1}, \ldots, i_{r}\right) \in \Gamma(T)} W\left(i_{1}, \ldots, i_{r}\right) .
$$

Это распределение совпадает с распределением случайной величины $\xi(s, T)$.

Мы воспользуемся оценкой локального варианта метода Чена - Стейна (см. [4], [5] и ссылки в этих работах). Пусть

$$
\begin{gathered}
\Gamma_{\left(i_{1}, \ldots, i_{r}\right)}^{i n d}(T)= \\
=\left\{\left(i_{1}^{\prime}, \ldots, i_{r}^{\prime}\right) \in \Gamma(T): \min \left\{\left|i_{k}^{\prime}-i_{k}\right|,\left|m_{k}-\left(i_{k}^{\prime}-i_{k}\right)\right|\right\} \geq s, k=1, \ldots, r\right\} .
\end{gathered}
$$

Положим

$$
\Gamma_{\left(i_{1}, \ldots, i_{r}\right)}(T)=\left(\Gamma(T) \backslash\left\{\left(i_{1}, \ldots, i_{r}\right)\right\}\right) \backslash \Gamma_{\left(i_{1}, \ldots, i_{r}\right)}^{i n d}(T) .
$$

Нетрудно проверить, что случайная величина $W\left(i_{1}, \ldots, i_{r}\right)$ не зависит от совокупности случайных величин

$$
\left\{W\left(i_{1}^{\prime}, \ldots, i_{r}^{\prime}\right):\left(i_{1}^{\prime}, \ldots, i_{r}^{\prime}\right) \in \Gamma_{\left(i_{1}, \ldots, i_{r}\right)}^{i n d}(T)\right\} .
$$

Поэтому оценка по методу Чена - Стейна (см., например, теорему 6.1 в [5]) принимает вид

$$
\rho\left(\mathcal{L}\left(\xi^{\prime}(s, T)\right), \operatorname{Po}(\Lambda)\right) \leq
$$




$$
\begin{gathered}
\leq \frac{1-e^{-\Lambda}}{\Lambda}\left(\sum_{\left(i_{1}, \ldots, i_{r}\right) \in \Gamma(T)} \mathbf{E} W\left(i_{1}, \ldots, i_{r}\right)\left(\mathbf{E} W\left(i_{1}, \ldots, i_{r}\right)+\mathbf{E} U\left(i_{1}, \ldots, i_{r}\right)\right)+\right. \\
\left.\quad+\sum_{\left(i_{1}, \ldots, i_{r}\right) \in \Gamma(T)} \mathbf{E} W\left(i_{1}, \ldots, i_{r}\right) U\left(i_{1}, \ldots, i_{r}\right)\right)
\end{gathered}
$$

где

$$
U\left(i_{1}, \ldots, i_{r}\right)=\sum_{\left(i_{1}^{\prime}, \ldots, i_{r}^{\prime}\right) \in \Gamma_{\left(i_{1}, \ldots, i_{r}\right)}(T)} W\left(i_{1}^{\prime}, \ldots, i_{r}^{\prime}\right) .
$$

Лемма 1. Пусть $2 s-1 \leq \min \left\{m_{1}, \ldots, m_{r}\right\}$. Тогда

$$
\begin{gathered}
\left|\left\{\left(i_{1}, \ldots, i_{r}\right)\right\} \cup \Gamma_{\left(i_{1}, \ldots, i_{r}\right)}(T)\right|=\left|\Gamma(T) \backslash \Gamma_{\left(i_{1}, \ldots, i_{r}\right)}^{i n d}(T)\right| \leq \\
\leq(2 s-1)(\rfloor \frac{T}{m_{1}}[+\ldots+] \frac{T}{m_{r}}[) .
\end{gathered}
$$

Доказательство. Проекции множества $\left\{\left(i_{1}, \ldots, i_{r}\right)\right\} \cup \Gamma_{\left(i_{1}, \ldots, i_{r}\right)}(T)$ на «окружности» $\left\{0, \ldots, m_{1}-1\right\}, \ldots,\left\{0, \ldots, m_{r}-1\right\}$ представляют собой отрезки из $2 s-1$ подряд идущих точек. Последовательность $t\left(m_{1}\right)$ с ростом параметра $t$ от 0 до $T-1$ проходит все точки множества $\left\{0, \ldots, m_{1}-1\right\}$ подряд (по кругу) не более $] T / m_{1}\left[\right.$ раз. В этом процессе значение $t\left(m_{1}\right)$ попадет на отрезок из $2 s-1$ точек не более $(2 s-1)] T / m_{1}[$ раз. Это число является оценкой сверху для числа тех элементов множества $\left\{\left(i_{1}, \ldots, i_{r}\right)\right\} \cup \Gamma_{\left(i_{1}, \ldots, i_{r}\right)}(T)$, которые попали в это множество за счет нарушения условия $\min \left\{\left|i_{1}^{\prime}-i_{1}\right|,\left|m_{1}-\left(i_{1}^{\prime}-i_{1}\right)\right|\right\} \geq s$. То же самое выполнено для остальных проекций. Из этих оценок следует неравенство (13). Лемма доказана.

Продолжим доказательство теоремы. Из определений вытекает, что при всех $\left(i_{1}, \ldots, i_{r}\right) \in \Gamma(T)$

$$
\mathbf{E} W\left(i_{1}, \ldots, i_{r}\right)=\frac{1}{M^{s}} .
$$

Из (13) и (14) следует, что

$$
\begin{aligned}
\mathbf{E} W\left(i_{1}, \ldots, i_{r}\right)+ & \mathbf{E} U\left(i_{1}, \ldots, i_{r}\right)=\left|\left\{\left(i_{1}, \ldots, i_{r}\right)\right\} \cup \Gamma_{\left(i_{1}, \ldots, i_{r}\right)}(T)\right| \frac{1}{M^{s}} \leq \\
& \leq \frac{2 s-1}{M^{s}}(] \frac{T}{m_{1}}[+\ldots+] \frac{T}{m_{r}}\lceil)
\end{aligned}
$$


Поэтому первая сумма в правой части (11) допускает оценку

$$
\begin{gathered}
\sum_{\left(i_{1}, \ldots, i_{r}\right) \in \Gamma(T)} \mathbf{E} W\left(i_{1}, \ldots, i_{r}\right)\left(\mathbf{E} W\left(i_{1}, \ldots, i_{r}\right)+\mathbf{E} U\left(i_{1}, \ldots, i_{r}\right)\right) \leq \\
\leq \frac{\Lambda(2 s-1)}{M^{s}}(\rfloor \frac{T}{m_{1}}[+\ldots+\rceil \frac{T}{m_{r}}\lceil) .
\end{gathered}
$$

Оценим вторую сумму в правой части (11).

Лемма 2. Пусть $2 s-1 \leq \min \left\{m_{1}, \ldots, m_{r}\right\}$,

$$
\left(i_{1}^{\prime}, \ldots, i_{r}^{\prime}\right) \in \Gamma_{\left(i_{1}, \ldots, i_{r}\right)}(T), \quad\left(i_{1}, \ldots, i_{r}\right) \in \Gamma(T) .
$$

Тогда $\mathbf{E} W\left(i_{1}, \ldots, i_{r}\right) W\left(i_{1}^{\prime}, \ldots, i_{r}^{\prime}\right) \leq M^{1-2 s}$.

K лемме 2 мы вернемся в конце статьи, а пока продолжим доказательство теоремы. Согласно (12), (13) и оценке леммы 2

$$
\begin{gathered}
\left.\sum_{\left(i_{1}, \ldots, i_{r}\right) \in \Gamma(T)} \mathbf{E W (} i_{1}, \ldots, i_{r}\right) U\left(i_{1}, \ldots, i_{r}\right)<\sum_{\left(i_{1}, \ldots, i_{r}\right) \in \Gamma(T)}\left|\Gamma_{\left(i_{1}, \ldots, i_{r}\right)}(T)\right| \frac{1}{M^{2 s-1}} \leq \\
\leq \frac{\Lambda(2 s-1)}{M^{s-1}}(] \frac{T}{m_{1}}[+\ldots+] \frac{T}{m_{r}}\lceil) .
\end{gathered}
$$

Из (11), (15) и (16) следует, что

$$
\begin{gathered}
\rho\left(\mathcal{L}\left(\xi^{\prime}(s, T)\right), \operatorname{Po}(\Lambda)\right) \leq \\
\leq \frac{(M+1)(2 s-1)}{M^{s}}(\rfloor \frac{T}{m_{1}}[+\ldots+] \frac{T}{m_{r}}\lceil) .
\end{gathered}
$$

Из (17) получаем неравенство (5). Теорема доказана.

Доказательство следствий 1 и 2. Из (4), неравенства $T \leq m_{1} \ldots m_{r}$ и условия $\Lambda \rightarrow \lambda \in(0, \infty]$ следует, что $s=O\left(\ln m_{1}+\ldots+\ln m_{r}\right)$, а правая часть в (5) допускает при переходе к пределу оценку

$$
O\left(\frac{s T}{M^{s}}\left(\frac{1}{m_{1}}+\ldots+\frac{1}{m_{r}}\right)\right)=O\left(\Lambda \max _{i, j}\left(\frac{\ln m_{i}}{m_{j}}\right)\right) .
$$

Поэтому по условиям следствий она стремится к нулю, и из условия $\Lambda \rightarrow \lambda \in(0, \infty)$ и теоремы 1 вытекает сходимость распределения величины $\xi(s, T)$ к распределению Пуассона Ро $(\lambda)$. Следствие 1 доказано. 
В свою очередь, в случае $\Lambda \rightarrow \infty$ из сходимости к нулю выражений (18) и теоремы 1 вытекает сходимость к нулю расстояния по вариации между распределением случайной величины $\xi(s, T)$ и распределением Пуассона $\operatorname{Po}(\Lambda)$ с растущим к бесконечности параметром $\Lambda$. Последнее распределение при указанных в формулировке центрировке и нормировке стремится к стандартному нормальному распределению. Поэтому выполнено утверждение следствия 2. Итак, оба следствия доказаны.

Доказательство леммы 2. Пусть $1 \leq t<t+l \leq m_{1} \ldots m_{r}-1$. Событие $B_{t} \cap B_{t+l}$ можно трактовать как выполнение системы из $2 s$ сравнений

$$
\begin{gathered}
X_{t\left(m_{1}\right)}^{(1)}+\ldots+X_{t\left(m_{r}\right)}^{(r)} \equiv a_{t} \quad \bmod M, \quad t=1, \ldots, s, \\
X_{(t+l)\left(m_{1}\right)}^{(1)}+\ldots+X_{(t+l)\left(m_{r}\right)}^{(r)} \equiv a_{t} \bmod M, \quad t=1, \ldots, s .
\end{gathered}
$$

Система (19) - (20) обладает следующими свойствами.

1) Согласно условию $2 s-1 \leq \min \left\{m_{1}, \ldots, m_{r}\right\}$ множества неизвестных в уравнениях каждой из систем (19) и (20) не пересекаются.

2) Комбинация, выражающая уравнение системы (19) через уравнения системы (20), если она существует, может быть получена лишь из одного уравнения системы (20) (иначе в ней оказалось бы более $r$ ненулевых слагаемых). То же самое верно и для уравнения системы (20). А значит, возможны лишь следующие варианты:

$2 \mathrm{a})$ системы (19) и (20) имеют общее уравнение,

2б) ни одно уравнение системы (19) не может быть выражено через уравнения системы (20), и обратно, ни одно уравнение системы (20) не может быть выражено через уравнения системы (19) - (20).

В случае $2 \mathrm{a})$ события $B_{t}$ и $B_{t+l}$ несовместны, так как цепочка $a_{1}, \ldots, a_{s}$ не может наложиться сама на себя. Следовательно (здесь и ниже в $(22)$ $\left.i_{k}=t\left(m_{k}\right), i_{k}^{\prime}=(t+l)\left(m_{k}\right), k=1, \ldots, r\right)$,

$$
\mathbf{E} W\left(i_{1}, \ldots, i_{r}\right) W\left(i_{1}^{\prime}, \ldots, i_{r}^{\prime}\right)=0 .
$$

В случае 2б) каждое следующее уравнение системы (19) - (20) (уравнения можно брать в любом порядке) содержит хотя бы одно неизвестное, не входящее в предыдущие уравнения. Это означает, что, по крайней мере, $2 s-1$ неизвестных системы (19) - (20) однозначно определяются остальными неизвестными. Следовательно,

$$
\mathbf{E} W\left(i_{1}, \ldots, i_{r}\right) W\left(i_{1}^{\prime}, \ldots, i_{r}^{\prime}\right)<\mathbf{P}\left\{B_{t} \cap B_{t^{\prime}}\right\} \leq M^{1-2 s} .
$$

Из (21) и (22) вытекает, что неравенство (22) выполнено в обоих случаях. Лемма 2 доказана. 
Автор признателен А. М. Зубкову за полезные замечания.

\section{Список литературы}

[1] Pohl P., "Description of MCV, a pseudo-random number generator", Scand. Actuarial J., 1976, № 1, 1-14.

[2] Меженная Н. М., Михайлов В. Г., “Оценки и предельные теоремы нормального типа для числа единиц в выходной последовательности генератора Пола", Математические вопросы криптографии, 4:4 (2013), 95-107.

[3] Chryssaphinou O., Papastavridis S., "A limit theorem for the number of non-overlapping occurrence of a pattern in a sequence of independent trials", J. Appl. Prob., 25:2 (1988), 428-431.

[4] Barbour A. D., Holst L., Janson S., Poisson Approximation, Oxford: Oxford University Press, 1992.

[5] Михайлов В. Г., "Явные оценки в предельных теоремах для сумм случайных индикаторов", Обозр. прикл. и промыил. матем., 1:4 (1994), 580-617. 